\section{Cuidado mãe canguru em recém-nascidos pré-termo sob suporte ventilatório: avaliação dos estados comportamentais}

\section{Kangaroo mother care in preterm newborns on artificial ventilation: an evaluation of behavior patterns}

Vívian Mara Gonçalves de Oliveira Azevedo 1 Renata Bernardes David 2

César Coelho Xavier 3
1,2 Hospital Sofia Feldman. Rua Antônio Bandeira, 1060. Bairro Tupi. Belo Horizonte, MG, Brasil. CEP: 31.844-130.

Email: viviangazevedo@hotmail.com

3 Departamento de Pediatria. Faculdade de Medicina. Universidade Federal de Minas Gerais. Belo Horizonte, MG, Brasil.

\begin{abstract}
Objectives: to describe the behavior of newborns weighing under $1500 \mathrm{~g}$ on mechanical ventilation, before and after kangaroo mother care (KMC).

Methods: a quasi-experimental type study in which behaviors such as deep sleep, activity, drowsiness, inactive alertness, active alertness, and crying as defined by the Brazelton Neonatal Scale were assessed on three separate occasions: 15 minutes before KMC, 30 after the beginning of KMC and 15 minutes after a total of one hour's KMC. Forty-four preterm newborns (statistical power > 0.9), with a mean gestational age of 29 weeks and mean weight of $1096 \mathrm{~g}$, intubated and stable in terms of hemodynamics were selected by way of continuous entry sampling.

Results: the results provided evidence that KMC improves babies' sleep, especially deep sleep $(52.3 \%)$, compared to the period prior to (6.8\%) and after $(13.6 \%)(p<0.001)$ KMC. Apart from this, the newborns showed signs of pain after $K M C$, with a significant difference $(p=0.002)$.

Conclusions: kangaroo mother care, under the conditions studied, may be considered a beneficial strategy for neuro-behavioral development.
\end{abstract}

Key words Premature, Child development, Kangaroo mother method

\section{Resumo}

Objetivos: descrever os estados comportamentais dos recém-nascidos com peso inferior a 1500 $g$, em ventilação mecânica, antes, durante e após o cuidado mãe canguru (CMC).

Métodos: estudo do tipo quase experimental em que os estados comportamentais definidos como sono profundo, ativo, sonolência, alerta inativo, alerta ativo e choro de acordo com a Escala Neonatal de Brazelton foram avaliados em três momentos: 15 minutos antes do CMC, 30 minutos após o início da exposição e 15 minutos após o CMC num total de uma hora de exposição. Quarenta e quatro recém-nascidos pré-termo (poder estatístico >0,9), com idade gestacional média de 29 semanas e peso médio de $1096 \mathrm{~g}$, intubados e estáveis do ponto de vista hemodinâmico, foram selecionados por amostra de entrada contínua.

Resultados: evidenciou-se o favorecimento do sono, principalmente o sono profundo $(52,3 \%)$, comparado aos periodos antes $(6,8 \%)$ e após $(13,6 \%)(p<0,001)$ o CMC. Além disso, os recémnascidos apresentaram sinais de dor após o CMC com diferença significativa $(p=0,002)$.

Conclusões: o cuidado mãe canguru, nas condições deste estudo, pode ser considerado como uma estratégia favorecedora para o desenvolvimento neurocomportamental.

Palavras-chave Prematuro, Desenvolvimento infantil, Método mãe canguru 


\section{Introdução}

O cuidado mãe canguru (CMC), criado por Edgar Rey Sanabria em 1978 na Colômbia, consiste no contato pele a pele precoce, entre mãe e filho. Esta técnica é usada como incentivadora da participação dos pais e da família no cuidado aos filhos prematuros e de baixo peso. Além disso, representa uma alternativa na humanização do cuidado neonatal, pela promoção do vínculo mãe-filho e do aleitamento materno, dois elementos essenciais para a sobrevida e o favorecimento do desenvolvimento dos recém-nascidos $(\mathrm{RN})$ vulneráveis. ${ }^{1}$

Além de proporcionar oportunidades de interação mãe-filho, ${ }^{2}$ o CMC favorece o desenvolvimento neurocomportamental, pois oferece estímulos olfatórios, auditivos, tácteis, térmicos e proprioceptivos.3,4 VandenBerg, 5 ressalta que a incidência de alterações no desenvolvimento neurocomportamental entre os prematuros permanece alta e problemática. Dentre os fatores que contribuem para essas alterações, estão o baixo peso ao nascer e o estresse provocado pelo ambiente da Unidade de Terapia Intensiva Neonatal (UTIN). O sistema nervoso imaturo dos RNs pré-termo pode ser insuficiente para lidar com os estímulos nociceptivos e a compreensão de suas respostas comportamentais é essencial para que se tenha uma boa qualidade na assistência.

A maturidade do sistema nervoso dos RN expostos a diferentes níveis de riscos clínicos 6 pode ser determinada por meio do tônus vagal, da organização dos estados comportamentais e do status neurocomportamental, também utilizados na predição da função cognitiva e social.7,8 Os estados comportamentais, descritos por Brazelton 9 e Holditch-Davis, 10 são considerados tópicos importantes do exame comportamental e para a compreensão das reações dos RN.

Frequentemente, os estudos sobre o CMC avaliam o comportamento dos $\mathrm{RN}$ estáveis sem necessidade de oxigenoterapia.2,4,11-14 Contudo, há evidências que recomendam a estratégia mesmo em RN criticamente enfermos e com suporte ventilatório. 15,16 Ludington-Hoe et al. 15 afirmam que o $\mathrm{CMC}$ pode ser benéfico mesmo em RN com oxigenoterapia, pois a posição vertical aumenta a eficiência do diafragma e da função pulmonar, favorecendo a oxigenação e promovendo estabilidade cardiorespiratória. Van Zanten et al.,16 estudaram prematuros com idade gestacional (IG) inferior a 30 semanas e peso médio de $1044 \mathrm{~g}$ sob suporte ventilatório e observaram que o CMC pode ser seguro, apesar da importância de se mensurar a temperatura corporal de $\mathrm{RN}$ constantemente. No entanto, no estudo de Smith, 17 realizado com 14 RN prematuros com diagnóstico de doença pulmonar crônica, em ventilação mecânica (VM), foi observada instabilidade nas variáveis fisiológicas descritas durante o $\mathrm{CMC}$, realizado por duas horas.

O CMC pode favorecer a maturação do sistema nervoso autônomo e do ritmo circadianos ${ }^{3}$ reduzir o tempo de choro 18 e estimular a amamentação. ${ }^{11}$ Além disso, estudos apontam que os resultados do CMC sobre os RNs pré-termo podem se prolongar após o período neonatal, proporcionando, na idade do termo, maior organização no ciclo sono-vigília, maior capacidade de adaptação aos estímulos aversivos aos três meses de idade e maior competência na exploração de objetos durante o brincar aos seis meses de idade, quando comparados aos respectivos pares do grupo controle. 4,12

O objetivo deste estudo foi investigar os estados comportamentais dos $\mathrm{RN}$ com peso inferior a 1500 $\mathrm{g}$, em ventilação mecânica, no cuidado mãe canguru.

\section{Métodos}

Trata-se de um estudo do tipo quase-experimental, onde os RNs foram avaliados quanto aos estados comportamentais segundo Brazelton, ${ }^{9}$ antes, durante e após a exposição ao CMC. As medidas iniciais serviram como controle das medidas subsequentes. O estudo foi conduzido no período de agosto de 2007 a fevereiro de 2008, na UTIN do Hospital Sofia Feldman - instituição filantrópica ligada ao Sistema Único de Saúde e localizada na periferia de Belo Horizonte, Minas Gerais, Brasil.

O cálculo amostral foi realizado considerando-se a diferença entre o maior e o menor valor das variáveis quantitativas avaliadas quanto à segurança do CMC (frequência cardíaca, pressão arterial, temperatura corporal e a saturação de oxigênio) estudada nesta linha de pesquisa, em situação pareada. 19 A diferença dos valores dessas variáveis indicou que o tamanho amostral de $44 \mathrm{RN}$ correspondeu a um poder acima de 0,90 e a um nível de significância de 0,05 . As análises foram feitas utilizando o software $\mathrm{R}$.

Foram elegíveis $54 \mathrm{RN}$ intubados, estáveis dos pontos de vista respiratório e hemodinâmico por pelo menos 24 horas. Os seguintes critérios de exclusão foram adotados no desenho do estudo: $\mathrm{RN}$ com níveis elevados de bilirrubina e que necessitavam de fototerapia, RN de mulheres com alterações de pele que inviabilizassem o posicionamento canguru e aqueles com malformações congênitas ou com diagnóstico de hemorragia intraventricular de graus III e 
IV. Nenhum dos RN elegíveis preencheu qualquer um dos critérios de exclusão. Dos 54 RN, 10 $(18,5 \%)$ não participaram devido à recusa materna.

A exposição ao CMC teve a duração de uma hora e o RN, vestido apenas com fralda, foi colocado sobre o peito da mãe, em posição prono e vertical. A mãe, vestida com roupa apropriada (camisola com abertura anterior) era sentada em uma cadeira confortável e reclinável a $60^{\circ}$. O circuito do respirador foi afixado ao ombro da mãe com fitas adesivas. O procedimento foi realizado de acordo com o protocolo modificado de Ludington-Hoe et al. 15

O RN permanecia uma hora no CMC desde que os dados vitais (frequência cardíaca, temperatura corporal e saturação periférica de oxigênio) mantivessem seus valores dentro dos limites da normalidade. Não houve nenhum caso de instabilidade durante o posicionamento, que tornasse necessário o retorno à incubadora. Previamente foram apresentados resultados referentes à segurança do método, quando realizado com crianças de muito baixo peso e que necessitassem de suporte ventilatório. 20,21

A variável estado comportamental foi avaliada a partir da ocorrência dos estados comportamentais definidos em (1) sono profundo; (2) sono ativo; (3) sonolência; (4) alerta inativo; (5) alerta ativo; e (6) choro, de acordo com a Escala Neonatal de Avaliação de Brazelton em três momentos: a) 15 minutos antes do CMC; b) 30 minutos após o início da exposição; e c) 15 minutos após o CMC. Os registros da avaliação dos sinais comportamentais foram realizados por duas autoras (VMGOA e RBD) que obtiveram índice excelente de confiabilidade entre examinadores $($ Kappa $=1,0 ;$ IC $95 \%=0,49 \mathrm{a}$ $1,0)$.

Para a avaliação da dor, foi utilizado o Sistema de Codificação da Atividade Facial (NFCS). ${ }^{22}$ Esta escala considera a presença ou ausência dos seguintes movimentos faciais: fronte saliente, olhos cerrados, sulco naso-labial aprofundado, boca esticada, lábios entreabertos, lábios franzidos, língua tensa e tremor do queixo. Para cada um dos itens, quando presente, foi atribuído um ponto, sendo o escore máximo de oito pontos. Considera-se a presença de dor quando a pontuação é superior a dois. Para o estudo foram utilizados como ausência de dor $<3$ e a presença de dor $\geq 3$.

A análise dos dados foi feita por frequência e porcentagens das características das variáveis e da obtenção de medidas de tendência central (média) e medidas de dispersão (desvio-padrão). Os escores obtidos através da escala comportamental foram comparados nos tempos antes, durante e após o CMC, sendo aplicado o teste qui-quadrado de Pearson para a comparação de proporções e quando uma das frequências esperadas foi menor que cinco, utilizou-se o teste exato de Fisher. Para avaliação dos sinais de dor, analisada por meio da escala NFCS antes, durante e após o CMC, foi utilizado o teste exato de Fisher. As temperaturas do ambiente, da incubadora e da mãe foram medidas antes e após o CMC sendo aplicado o teste $t$ pareado.

A investigação foi aprovada pelos Comitês de Ética em Pesquisa da Universidade Federal de Minas Gerais ( $\left.\mathrm{n}^{\circ} 311 / 07\right)$ e do Hospital Sofia Feldman ( ${ }^{\circ}$ 07/2007).

\section{Resultados}

Dos $44 \mathrm{RN}$ incluídos no estudo, 57\% eram do sexo masculino e $95 \%$ tinham o diagnóstico de Síndrome do Desconforto Respiratório. A idade gestacional (IG) média ao nascimento foi de $29,1 \pm 1,6$ semanas e durante a intervenção de 7,0 \pm 7,0 dias de vida. $O$ peso médio ao nascer foi de $1132,6 \pm 229,1$ gramas e, no momento da intervenção, de 1098,0 0247,0 gramas e $93 \%$ tinham peso adequado para a idade gestacional.23

Em relação aos estados comportamentais, observa-se na Tabela 1, que o CMC favoreceu o sono, principalmente o profundo e que $52,3 \%$ dos RNs permaneceram nesse estado, comparado aos períodos antes $(6,8 \%)$ e após $(13,6 \%)$.

A Tabela 2 apresenta os resultados para a avaliação da dor. Observa-se que os RN apresentaram sinais de dor após o $\mathrm{CMC}$ com diferença significativa $(p=0,002)$.

Em relação às variáveis de controle, os resultados revelaram que a temperatura axilar da mãe, do ambiente e da incubadora não apresentaram diferenças com significância estatística para as médias.

Todos os RN permaneceram estáveis (dados vitais dentro dos limites de normalidade) durante o posicionamento, não havendo necessidade de interrupção do CMC. 
Tabela 1

Distribuição dos estados comportamentais dos recém-nascidos segundo a exposição ao cuidado mãe canguru.

\begin{tabular}{|c|c|c|c|c|c|c|c|c|c|c|c|c|c|}
\hline \multirow{3}{*}{$\begin{array}{l}\text { Momentos da } \\
\text { avaliação }\end{array}$} & \multicolumn{12}{|c|}{ Estados comportamentais } & \multirow{3}{*}{$p$} \\
\hline & \multicolumn{2}{|c|}{ Alerta ativo } & \multicolumn{2}{|c|}{ Alerta inativo } & \multicolumn{2}{|c|}{ Choro } & \multicolumn{2}{|c|}{ Sono leve } & \multicolumn{2}{|c|}{ Sono profundo } & \multicolumn{2}{|c|}{ Sonolento } & \\
\hline & $\mathrm{n}$ & $\%$ & $\mathrm{n}$ & $\%$ & $\mathrm{n}$ & $\%$ & $\mathrm{n}$ & $\%$ & $\mathrm{n}$ & $\%$ & $\mathrm{n}$ & $\%$ & \\
\hline Antes & 17 & 38,6 & 1 & 2,2 & 0 & 0,0 & 14 & 31,8 & 3 & 6,8 & 9 & 20,5 & $<0,001$ * \\
\hline Durante CMC & 0 & 0,0 & 1 & 2,2 & 0 & 0,0 & 17 & 38,6 & 23 & 52,3 & 3 & 6,8 & \\
\hline Após & 13 & 29,5 & 2 & 4,6 & 3 & 6,8 & 14 & 31,8 & 6 & 13,6 & 6 & 13,6 & \\
\hline
\end{tabular}

CMC = cuidado mãe canguru; * Teste qui-quadrado.

Tabela 2

Distribuição dos escores da escala Neonatal Facial Coding System na avaliação da dor antes, durante e após o cuidado mãe canguru.

\begin{tabular}{|c|c|c|c|c|c|}
\hline \multirow[t]{3}{*}{ Momentos da avaliação } & \multicolumn{4}{|c|}{ Neonatal Facial Coding System } & \multirow{3}{*}{$p$} \\
\hline & \multicolumn{2}{|c|}{$<3$} & \multicolumn{2}{|c|}{$\geq 3$} & \\
\hline & $\mathrm{n}$ & $\%$ & $\mathrm{n}$ & $\%$ & \\
\hline Antes & 43 & 97,7 & 1 & 2,3 & \\
\hline \multicolumn{6}{|l|}{ Durante o CMC } \\
\hline 2 minutos & 44 & 100,0 & 0 & 0,0 & \\
\hline 20 minutos & 44 & 100,0 & 0 & 0,0 & \\
\hline 40 minutos & 43 & 97,7 & 1 & 2,3 & \\
\hline 60 minutos & 44 & 100,0 & 0 & 0,0 & \\
\hline Após & 38 & 86,4 & 6 & 13,6 & $0,002 *$ \\
\hline
\end{tabular}

$\mathrm{CMC}=$ cuidado mãe canguru; ${ }^{*}$ Teste Exato de Fisher .

\section{Discussão}

Os resultados mostraram que o CMC pode ser considerado uma estratégia favorecedora do desenvolvimento neurocomportamental, mesmo em RN sob suporte ventilatório, visto que favoreceu o estado de sono, principalmente o sono profundo, considerado fundamental para o desenvolvimento e a organização cerebral em RN pré-termo. ${ }^{24}$

Resultados semelhantes foram encontrados por Lundington-Hoe et al. $25 \mathrm{em}$ um estudo randomizado, envolvendo 28 RN pré-termo com IG inferior a 32 semanas. Foi observado, por meio de eletroencefalograma, que o grupo submetido ao CMC por até três horas, apresentou menor agitação durante todo o período do estudo quando comparado ao grupo que recebeu os cuidados na incubadora.

Do mesmo modo, no estudo de Bauer et al.,26 onde foram avaliados $27 \mathrm{RN}$ pré-termo, entre 25-30 semanas, respirando espontaneamente, observou-se maior duração de sono durante o CMC quando comparado ao grupo controle: $90 \%$ do tempo para os de IG entre $25-27$ semanas e $97 \%$ para os de IG entre 28-30 semanas.

O sono profundo é considerado extremamente necessário como favorecedor do desenvolvimento, inclusive cerebral, pois nesse estado há maior conservação de energia, especialmente para os RN pré-termo.27,28 De acordo com a recomendação para implementação do CMC em bebês pré-termo a partir 
de 30 semanas de IG, desenvolvido por LudingtonHoe et al.,29 o CMC pode ser utilizado como estratégia para o favorecimento do estado de sono.

Feldman et al. ${ }^{4}$ realizaram uma investigação com RNs pré-termo, com IG média de 30 semanas, submetidos ao CMC por uma hora diariamente, ao longo de 14 dias e mostraram que o procedimento foi suficiente para evidenciar maior período de sono profundo e menor tempo em sono ativo. Além disso, os RN submetidos ao $\mathrm{CMC}$ apresentaram organização madura do ciclo sono-vigília quando comparados àqueles não submetidos. Segundo os mesmos autores, o contato pele a pele com a mãe influenciou também na maturação autonômica e organizacional dos estados comportamentais de bebês, observados com 37 semanas de idade corrigida, avaliados a partir do ganho de tônus vagal e da melhora do status neurocomportamental, de acordo com a Escala Neonatal de Avaliação Comportamental de Brazelton. O aumento do tônus vagal resultou na habilidade dessas crianças para se organizarem frente aos estímulos externos e modularem os gastos energéticos, e o resultado da avaliação comportamental revelou que as crianças tinham nível de neurodesenvolvimento maduro e integrado.

Feldman e Eidelman 12 observaram ainda que os efeitos do CMC podem ser prolongados após o período neonatal, uma vez que o CMC teve resultado positivo em relação ao desenvolvimento motor e cognitivo dos bebês e na interação e percepção dos pais, referentes aos bebês com 37 semanas aos três e seis meses de idade cronológica.

A avaliação da dor antes, durante e após o CMC apresentou diferença significativa após a retirada do posicionamento, o que pode ter influenciado na avaliação dos estados comportamentais nesse momento. Não foi observado redução dos sinais de dor nas crianças nos períodos antes e durante o CMC. Outros estudos, entretanto, verificaram redução dos sinais de dor durante o CMC, especialmente durante os procedimentos dolorosos.30,31 Observou-se, no entanto, a presença significativa de sinais dolorosos após a retirada do $\mathrm{CMC}$, o que pode sugerir que este seria um sinal de desconforto pela retirada do bebê do contato pele a pele com a mãe.

Consideram-se como limitação do estudo a não alocação aleatória dos participantes e a dificuldade de se separarem os efeitos do CMC dos fatores externos ou de confusão. Outra limitação foi o tempo de avaliação dos $\mathrm{RN}$, realizada em apenas três momentos e durante uma hora de posicionamento. Há também restrição quanto à aplicação da Escala Neonatal de Avaliação Comportamental para prematuros antes de atingir a idade gestacional corrigida de 40 semanas. Estudos futuros são necessários, uma vez que os resultados deste trabalho não permitem generalizações e que investigações em populações muito vulneráveis necessitam de avaliações com o maior rigor metodológico possível.

Apesar das limitações metodológicas mencionadas neste estudo, realizado com $44 \mathrm{RN}$ prematuros, o CMC pode se constituir em estímulo ao desenvolvimento neurocomportamental, uma vez que favoreceu a ocorrência do sono profundo.

\section{Agradecimentos}

Agradecemos a todos os profissionais e ao Programa Incubadora da Integralidade do Hospital Sofia Feldman pelas contribuições à realização deste estudo.

\section{Referências}

1. Charpak N, Ruiz JG, Zupan J, Cattaneo A, Figueroa Z, Tessier R, Cristo M, Anderson G, Ludington S, Mendoza S Mokhachane M, Worku B. Kangaroo mother care: 25 years after. Acta Paediatr. 2005; 94: 514-22.

2. Cruvinel FG, Macedo EC. Interação mãe-bebê pré-termo e mudança no estado de humor: comparação do método mãecanguru com visita na unidade de terapia intensiva neonatal. Rev Bras Saúde Matern Infant. 2007; 7: 449-55.

3. Ludington-Hoe SM, Swinth JY. Developmental aspects of kangaroo care. J Obstet Gynecol Neonatal Nurs. 1996; 25: 691-703.

4. Feldman R, Weller A, Sirota L, Eidelman AI. Skin-to-skin contact (kangaroo care) promotes self-regulation in premature infants: sleep-wake cyclicity, arousal modulation, and sustained exploration. Dev Psychol. 2002; 38: 194-207.

5. VandenBerg KA. Individualized developmental care for high risk newborns in the NICU: a practice guideline. Early Hum Dev. 2007; 83: 433-42.

6. Als HA. Synactive model of neonatal behavior organization: framework for the assessment of neurobehavioral development in the premature infant and for support of infants and parents in the neonatal intensive care environment. Phys Occup Ther Pediatr. 1986; 6: 3-55.

7. Anders TF, Keener MA, Kraemer H. Sleep-wake state organization, neonatal assessment and development in premature infants during the first year of life. Sleep. 1985; 8: 193206.

8. Beckwith L, Parmelee AH. EEG patterns of preterm infants, home enviroment, and later IQ. Child Dev. 1986; 57: 77789. 
9. Brazelton TB. Neonatal behavioral assessment scale. London: William Heinemann Medical Books; 1973.

10. Holditch-Davis D. The development of sleeping and waking states in high-risk preterm infants. Infant Behav Dev. 1990; 13: 513-31

11. Andrade ISN, Guedes ZCF. Sucção do recém-nascido prematuro: comparação do método mãe-canguru com os cuidados tradicionais. Rev Bras Saúde Matern Infant. 2005; 5: 61-9.

12. Feldman R, Eidelman AI. Skin-to-skin contact (kangaroo care) accelerates autonomic and neurobehavioral maturation in premature infants. Dev Med Child Neurol. 2003; 45: $1-8$

13. Johnston CC, Stevens B, Pinelli J, Gibbins S, Filion F, Jack A, Steele S, Boyer K, Veilleus A. Kangaroo care is effective in diminishing pain response in preterm neonates. Arch Pediatr Adolesc Med. 2003; 157: 1084-8.

14. Ferber SG, Makhoul IR. The effect of skin-to-skin contact (kangaroo care) shortly after birth on the neurobehavioral responses of the term newborn: a randomized, controlled trial. Pediatrics. 2004; 113: 858-65.

15. Ludington-Hoe SM, Ferreira C, Swinth J, Ceccardi JJ. Safe criteria and procedure for kangaroo care with intubated preterm infants. J Obstet Gynecol Neonatal Nurs. 2003; 32: 579-88.

16. Van Zanten HA, Havenaar AJ, Stigt HJH, Ligthart PAH, Walther FJ. The kangaroo method is safe for premature infants under 30 weeks of gestation during ventilatory support. J Neonatal Nurs. 2007; 13: 186-90.

17. Smith SL. Physiologic stability of intubated VLBW infants during skin-to-skin care and incubator care. Adv Neonatal Care. 2001; 1: 28-40.

18. Moore ER, Anderson GC, Bergman N. Early skin-to-skin contact for mothers and their healthy newborn infants. The Cochrane Database Syst Rev. 2007; 18: CD003519.

19. Lemeshow S, Hosmer DW, Klar J, Lwanga SK. Adequacy of sample size in health studies. New York: The World Health Organization, John Wiley and Sons; 1990.

20. Nyqvist KH. Towards universal Kangaroo Mother Care: recommendations and report from the first european conference and seventh international workshop on kangaroo mother care. Acta Paediatr. 2010; 99: 820-6.

21. Azevedo VMGOA. Efeitos do cuidado mãe-canguru nos sinais vitais dos recém-nascidos prematuros com peso inferior a 1500 gramas em ventilação mecânica [dissertação].
Belo Horizonte: Faculdade de Medicina da Universidade Federal de Minas Gerais; 2008.

22. Grunau RVE, Craig KD. Pain expression in neonates. Pain. 1987; 28: 395-410.

23. Kramer MS, Platt RW, Wen SW, Joseph KS, Allen A, Abrahamowicz M, Blondel B, Bréart G. A new and improved population-based canadian reference for birth weight for gestational age. Pediatrics. 2001; 108: 1-7.

24. Messmer PR, Rodriguez S, Adam, J, Wells-Gentry J, Washburn K, Zabaleta I, Abreu S. Effect of kangaroo care on sleep time for neonates. Pediatr Nurs. 1997; 23: 408-14.

25. Lundington-Hoe SM, Johnson MW, Morgan K, Lewis T, Gutman J, Wilson PD, Scher MS. Neurophysiologic assessment of neonatal sleep organization: preliminary results of a randomized, controlled trial of skin contact with preterm infants. Pediatrics. 2006; 117: e909-23.

26. Bauer K, Pyper A, Sperling P, Uhrig C, Versmold H. Effects of gestational and postnatal age on body temperature, oxygen consumption, and activity during early skin-to-skin contact between preterm infants of 25-30-week gestation and their mothers. Pediatr Res. 1998; 44: 247-51.

27. Yecco GJ. Neurobehavioral development and developmental support of premature infants. J Perinatol Neonatal Nurs. 1993; 7: 56-65.

28. Hall D, Kirsten G. Kangaroo mother care: a review. Transfus Med. 2008; 18: 77-82.

29. Ludinton-Hoe SM, Morgan K, Abouelfettoh A. A clinical guideline for implementation of kangaroo care with premature infants of 30 or more weeks postmenstrual age. Adv Neonatal Care. 2008; 8: S3-S23.

30. Kostandy RR, Ludington-Hoe SM, Cong X, Abouelfettoh A, Bronson C, Stankus A, Jarrell JR. Kangaroo care (skin contact) reduces crying response to pain in preterm neonates: pilot results. Pain Manag Nurs. 2008; 9: 55-65.

31. Johnston CC, Filion F, Campbell-Yeo M, Goulet C, Bell L, McNaughton K, Byron J, Aita M, Finley GA, Walker CD. Kangaroo mother care diminishes pain from heel lance in very preterm neonates: a crossover trial. BMC Pediatr. 2008; 24: 8-13.

Recebido em 16 de março de 2010

Versão final apresentada em 17 de março de 2011

Aprovado em 30 de março de 2011 